SYMPLECTIC SINGULARITIES AND GEOMETRY OF GAUGE FIELDS

BANACH CENTER PUBLICATIONS, VOLUME 39

INSTITUTE OF MATHEMATICS

POLISH ACADEMY OF SCIENCES

WARSZAWA 1997

\title{
SYMPLECTIC CAPACITIES IN MANIFOLDS
}

\author{
ALFRED F. KÜNZLE \\ Département de Mathématiques, École Polytechnique Fédérale de Lausanne \\ 1015 Lausanne, Suisse \\ E-mail: kuenzle@masg1.epfl.ch
}

\begin{abstract}
Symplectic capacities coinciding on convex sets in the standard symplectic vector space are extended to any subsets of symplectic manifolds. It is shown that, using embeddings of non-smooth convex sets and a product formula, calculations of some capacities become very simple. Moreover, it is proved that there exist such capacities which are distinct and that there are star-shaped domains diffeomorphic to the ball but not symplectomorphic to any convex set.
\end{abstract}

1. Preliminaries. For an introduction to symplectic capacities, non-smooth Hamiltonian systems and characteristic differential inclusions we refer to a previous talk given at the Banach Center in October 93 [K93].

The aim of this note is to show that some calculations of symplectic capacities can be simplified through embeddings of non-smooth convex sets. No approximations by families of Hamiltonian functions are needed. We show that definitions of capacities of convex sets in the symplectic model space $\left(\mathbb{R}^{2 n}, \omega\right)$ suffice to define and to calculate in some cases symplectic capacities for subsets in any symplectic manifolds. Moreover, some applications of the product formula for convex sets derived in [K90] are given.

To define the setting, let us consider the standard symplectic linear space $V:=$ $\left(\mathbb{R}^{2 n}, \omega\right)$. The non-degenerate closed 2 -form $\omega$ is expressed by the almost complex structure $J_{0}: T \mathbb{R}^{2 n} \rightarrow T \mathbb{R}^{2 n}$, which is described in standard coordinates by an $n$-fold tensor product of the matrix $\left(\begin{array}{cc}0 & -1 \\ 1 & 0\end{array}\right) \cong i$. We write in these coordinates $x . y=\sum_{i=1}^{2 n} x_{i} y_{i}$ for the scalar product and $\omega(x, y)=J_{0} x . y$ for the symplectic form. A differentiable map $\varphi: V \rightarrow V$ is called symplectic if $\varphi^{*} \omega=\omega$, i.e. $d \varphi(x)^{T} J_{0} d \varphi(x)=J_{0}$. We denote the set of symplectic embeddings of open subsets of $\mathbb{R}^{2 n}$ into $\mathbb{R}^{2 n}$ by $\mathcal{E}_{\omega}\left(\mathbb{R}^{2 n}\right)$ and the symplectic diffeomorphisms of $\mathbb{R}^{2 n}$ by $\mathcal{D}_{\omega}\left(\mathbb{R}^{2 n}\right)$.

Let $B(r)=B^{2 n}(r)=\left\{x \in \mathbb{R}^{2 n}|| x \mid<r\right\}$ be the ball and $Z(r)=B^{2}(r) \times \mathbb{R}^{2 n-2}=$ $\left\{x \in \mathbb{R}^{2 n} \mid q_{1}^{2}+p_{1}^{2}<r^{2}\right\}$ be a cylinder with a symplectic base disc, where $p_{1}, q_{1}$ are the

1991 Mathematics Subject Classification: Primary 58F05; Secondary 52A20, 58C27, 58F22.

The paper is in final form and no version of it will be published elsewhere. 
first two coordinates.

Let $\mathcal{K}$ be the set of possibly unbounded convex sets with perhaps empty interior. Given such a convex set $K$, let $n_{K}(x)$ be the section of elements of length 1 in the normal cone (see e.g. [A84]) at a point $x$. We study the periodic characteristic differential inclusion of a non-smooth convex set $K$ which depends in fact only of the boundary of $K$ :

$$
\left.\begin{array}{cll}
\text { (i) } & \dot{\gamma}(t) \in J n_{K}(\gamma(t)) & \text { a.e. } \\
\text { (ii) } \gamma(t) \in \partial K & \forall t \in\left[0, T_{\gamma}\right] \\
\text { (iii) } \gamma\left(t+T_{\gamma}\right)=\gamma(t) & \forall t \in\left[0, T_{\gamma}\right] \\
\text { and } T_{\gamma}>0 \text { is the minimal period of } \gamma
\end{array}\right\}
$$

whose moduli space of solutions is called $\Gamma(K)$, which is in a well defined way equivalent to the periodic solutions of a non-smooth Hamiltonian system (see [K93]). The set of symplectic actions $A(\gamma)=\frac{1}{2} \int \dot{\gamma} \cdot J_{0} \gamma d t$ of elements of $\Gamma(K)$ is called the action spectrum of $K$.

DEFINITION 1. Let $c$ be the map

$$
\begin{aligned}
c: \mathcal{K} & \longrightarrow[0, \infty] \\
K & \longmapsto c(K)=\inf \{A(\gamma) \mid \gamma \in \Gamma(K)\}
\end{aligned}
$$

assigning to $K$ the minimal characteristic action of $\partial K$, using the convention that inf $=$ $\infty$ if $\Gamma(K)$ is empty.

It has been shown in [K90] that $c(K)$ (for a convex set $K$ with non-empty interior) can be expressed with a simple formula through the minimum of the classical dual Hamiltonian functional introduced by Clarke and Ekeland [CE80] and that it satisfies the axioms of a capacity of convex sets in the standard symplectic vector space. This means that $c$ coincides on smooth convex sets with the Ekeland-Hofer [EH89] and the Hofer-Zehnder capacity [HZ90] which are defined with the classical non-definite Hamiltonian functional and approximation by well chosen families of Hamiltonian functions. Moreover, $c$ satisfies a useful formula for symplectic products [K90] which we will use later: $c\left(K_{1} \times K_{2}\right)=\min \left\{c\left(K_{1}\right), c\left(K_{2}\right)\right\}$.

In this paper, we study the symplectic capacities extending $c$ :

Definition 2. Let $\mathcal{M}^{2 n}$ be the family of symplectic manifolds of given dimension $2 n$ and $\mathcal{S}$ a family of symplectic embeddings defined on open domains of such manifolds. Let further $\mathcal{F}$ be an $\mathcal{S}$-invariant family of subsets of these manifolds containing $\mathcal{K}$. We denote by $(D, \omega)$ the set $D$ with the symplectic form of the ambient manifold restricted to $D$ (which may be degenerate on $D$ ). A symplectic capacity for $\mathcal{F}$ and $\mathcal{S}$ extending $c$ is a map $C$ of $\mathcal{F}$ to $\mathbb{R}_{+}$satisfying

(a) $D, D^{\prime} \in \mathcal{F}, D \subset D^{\prime} \Longrightarrow C(D) \leq C\left(D^{\prime}\right)$,

(b) $D \in \mathcal{F}, \varphi \in \mathcal{S} \Longrightarrow C(\varphi(D))=C(D)$,

(c) if $K \in \mathcal{K}$, then $C(K)=c(K)$.

Capacities in $V$ are therefore obtained by taking $\mathcal{M}^{2 n}:=\left\{\mathbb{R}^{2 n}, \omega\right\}, \mathcal{F} \subset \mathcal{P}\left(\mathbb{R}^{2 n}\right)$, where $\mathcal{P}\left(\mathbb{R}^{2 n}\right)$ is the set of all subsets of $\mathbb{R}^{2 n}$, and we distinguish two cases: If $\mathcal{S}:=\mathcal{D}_{\omega}$ we call $C$ diffeomorphism capacity and if $\mathcal{S}:=\mathcal{E}_{\omega}$ we call it embedding capacity. 
The axioms are designed in the way that the existence of a symplectic capacity for $V$ implies Gromov's squeezing theorem: The existence of a symplectic embedding of the ball of radius $r$ into $Z(R)$ implies that $r \leq R$. However, to give a new proof of this theorem is not the aim of the present article.

2. Extensions in $\mathbb{R}^{2 n}$. In order to control all extensions of $c$ to any subset of $\mathbb{R}^{2 n}$ at the same time, the idea is to consider the smallest and biggest functions satisfying monotonicity and $\mathcal{D}_{\omega}$-invariance for $D \in \mathcal{P}\left(\mathbb{R}^{2 n}\right)$ :

\section{DEFINITION 3.}

$$
\begin{aligned}
& \ell(D)=\sup \left\{c(K) \mid K \in \mathcal{K} \quad \text { such that } \exists \varphi \in \mathcal{D}_{\omega} \text { with } \varphi(K) \subset D\right\} \\
& u(D)=\inf \left\{c(K) \mid K \in \mathcal{K} \quad \text { such that } \exists \varphi \in \mathcal{D}_{\omega} \text { with } D \subset \varphi(K)\right\} .
\end{aligned}
$$

Let analogously $\ell_{e}$ and $u_{e}$ be defined with symplectic embeddings $\varphi \in \mathcal{E}_{\omega}$ with open domain of definition $\operatorname{dom} \varphi \supset \bar{K}$ instead of diffeomorphisms $\mathcal{D}_{\omega}$. As usual, we set 0 the supremum and $\infty$ the infimum on the empty set.

We call $u$ and $\ell$ upper and lower symplectic capacity in $\mathbb{R}^{2 n}$ respectively because any capacity extending $c$ is estimated above and below by $u$ and $\ell$ :

\section{THEOREM 1.}

(i) All symplectic capacities $C: \mathcal{F} \rightarrow[0, \infty]$ coinciding on $\mathcal{K}$ with $c$ are estimated by $u$ and $\ell: \ell(D) \leq C(D) \leq u(D)$ for every $D \in \mathcal{F}$. If $C_{e}$ is moreover $\mathcal{E}_{\omega}$-invariant (an embedding capacity), it satisfies $\ell \leq \ell_{e} \leq C_{e} \leq u_{e} \leq u$.

(ii) $u$ and $\ell$ (and also $u_{e}$ and $\ell_{e}$ ) are symplectic diffeomorphism capacities for $\mathcal{P}\left(\mathbb{R}^{2 n}\right)$. $\ell_{e}$ is moreover an embedding capacity, whereas $u_{e}$ is not $\mathcal{E}_{\omega}$-invariant.

(iii) They all coincide on $\mathcal{K}$ with $c$;

(iv) $u$ and $\ell$ are distinct,

(v) and $u(D)=\inf _{\varphi \in \mathcal{D}_{\omega}} c(\operatorname{conv} \varphi(D))$, where conv $D$ is the closed convex hull of $D$.

Notation. We denote inward and outward approximation sets by

$$
\begin{aligned}
& \mathcal{I}(D)=\left\{K \in \mathcal{K} \mid \exists \varphi \in \mathcal{D}_{\omega} \quad \text { with } \varphi(K) \subset D\right\} \\
& \mathcal{O}(D)=\left\{K \in \mathcal{K} \mid \exists \varphi \in \mathcal{D}_{\omega} \quad \text { with } D \subset \varphi(K)\right\},
\end{aligned}
$$

then the proofs for $u$ and $\ell$ can simply be deduced from the properties of these sets.

Proof.

(i) We show only $\ell \leq C$. If $\ell=0$, there is nothing to prove since any capacity $C$ is non-negative. We may therefore suppose that there is $K \in \mathcal{K}$ and $\varphi \in \mathcal{D}_{\omega}$ with $\varphi(K) \subset D ;$ then

$$
C(D) \stackrel{(a)}{\geq} C(\varphi(K)) \stackrel{(b)}{=} C(K) \stackrel{(c)}{=} c(K),
$$

therefore $C(D) \geq \sup c(K)=\ell(D)$. An analogous argument yields $u(D) \geq C(D)$. The other inequalities can be proved in a similar way. 
(ii) Monotonicity: $D_{1} \subset D_{2} \Longrightarrow \mathcal{I}\left(D_{1}\right) \subset \mathcal{I}\left(D_{2}\right), \mathcal{O}\left(D_{1}\right) \supset \mathcal{O}\left(D_{2}\right)$, therefore

$$
\begin{aligned}
& \ell\left(D_{1}\right)=\sup _{\mathcal{I}\left(D_{1}\right)} c \leq \sup _{\mathcal{I}\left(D_{2}\right)} c=\ell\left(D_{2}\right) \\
& u\left(D_{1}\right)=\inf _{\mathcal{O}\left(D_{1}\right)} c \leq \inf _{\mathcal{O}\left(D_{2}\right)} c=u\left(D_{2}\right) .
\end{aligned}
$$

Symplectic invariance: Let $\psi \in \mathcal{D}_{\omega}$. For $K \in \mathcal{I}(\psi(D))$, there is $\varphi(K) \subset \psi(D) \Longrightarrow$ $\psi^{-1} \circ \varphi(K) \subset D \Longrightarrow K \in \mathcal{I}(D)$, by the group property of $\mathcal{D}_{\omega}$, thus $\mathcal{I}(\psi(D))=\mathcal{I}(D)$. Analogously, $\mathcal{O}(\psi(D))=\mathcal{O}(D)$, from where

$$
\begin{aligned}
& \ell(\psi(D))=\ell(D) \\
& u(\psi(D))=u(D) .
\end{aligned}
$$

The function $u_{e}(D):=\inf \left\{c(K) \mid K \in \mathcal{K}\right.$ such that $\exists \varphi \in \mathcal{E}_{\omega}$ with $\left.D \subset \varphi(K)\right\}$ satisfies immediately $u_{e}(D) \leq u(D)$. But $u_{e}$ is not $\mathcal{E}_{\omega}$-invariant (only $\mathcal{D}_{\omega}$-invariant):

$$
\psi(D) \subset \varphi(K) \quad \psi, \varphi \in \mathcal{E}_{\omega} \nRightarrow D \subset \psi^{-1} \circ \varphi(K)
$$

as $\psi^{-1}$ may not be defined on $\varphi(K)$. But $\ell_{e}$ is $\mathcal{E}_{\omega}$-invariant:

$$
\varphi(K) \subset \psi(D) \quad \psi, \varphi \in \mathcal{E}_{\omega} \Longrightarrow \psi^{-1} \varphi(K) \subset D
$$

since $\psi^{-1}$ is defined on the (smaller) set $\varphi(K)$.

(iii) To show $\ell(K)=c(K)=u(K)$ for all $K \in \mathcal{K}$, first note that

$$
\ell(K) \geq c(K) \geq u(K)
$$

because we can take $\varphi=i d$ in the definition of $\ell$ and $u$. For the reverse inequality, we need the monotonicity of a symplectic capacity on smooth convex domains such as $c_{E H}$ : For all $\varphi\left(K_{1}\right) \subset K \subset \psi\left(K_{2}\right)$ one gets $c\left(K_{1}\right) \leq c(K) \leq c\left(K_{2}\right)$ and therefore the claim by taking the infimum respectively the supremum on $K_{i}$.

(iv) We prove this by exhibiting an example: Consider the shell $A^{2 n}=B(R) \backslash B(r)$, $r<R$. To calculate $u\left(A^{2 n}\right)$, observe that all images of convex sets by diffeomorphisms containing $A^{2 n}$ contain $B(R)$, which is itself convex; therefore $u(A)=c(B(R))=\pi R^{2}$. For $\ell$, look first at an area-preserving embedding $\varphi_{0} \in \mathcal{E}_{\omega}$ in 2 dimensions $\varphi_{0}: K:=$ $(0,2 \pi) \times\left(0, \frac{R^{2}-r^{2}}{2}\right) \longrightarrow A^{2}$. Its image $\AA^{2} \backslash\{(p, q) \mid p=0, q>0\}$ has the same area as $K$ :

$$
c(K)=\pi\left(R^{2}-r^{2}\right)=\ell_{e}\left(\varphi_{0}(K)\right),
$$

and fills out $B(R) \backslash B(r)$ with respect to the area measure. Therefore, the lower embedding capacity $\ell_{e}\left(A^{2}\right):=\sup \left\{c(K) \mid K \in \mathcal{K}\right.$ such that $\exists \varphi \in \mathcal{E}_{\omega}$ with $\left.\varphi(K) \subset A^{2}\right\}$ equals $\pi\left(R^{2}-r^{2}\right)$. But $\ell\left(A^{2}\right)$ is less than $\ell_{e}\left(A^{2}\right)$ because $\mathcal{D}_{\omega} \subset \mathcal{E}_{\omega}$, from where we get the claim for dimension 2 :

$$
\ell\left(A^{2}\right) \leq \ell_{e}\left(A^{2}\right)=\pi\left(R^{2}-r^{2}\right)<\pi R^{2}=u\left(A^{2}\right) .
$$

The product formula for the symplectic product $P=A^{2} \times \cdots \times A^{2}$ yields finally $\ell(P)<u(P)$ for arbitrary dimensions. 


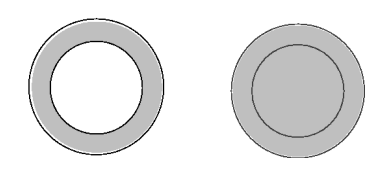

Fig. 1. Existence of distinct symplectic capacities.

(v) $\inf _{\varphi \in \mathcal{D}_{\omega}} c(\operatorname{conv} \varphi(D))=\inf _{\varphi} \inf _{K \in \mathcal{K}}\{c(K) \mid \varphi(D) \subset K\}$ by the definition of the convex hull and monotonicity for convex sets. This is equal to $\inf _{\varphi} \inf _{K}\{c(K) \mid D \subset$ $\left.\varphi^{-1}(K)\right\}=u(D)$.

Remark. To complete the calculation for the example in (iv), consider an areapreserving diffeomorphism $\psi_{\varepsilon} \in \mathcal{E}_{\omega}$ :

$$
\psi_{\varepsilon}: \stackrel{\circ}{A}^{2}=\stackrel{\circ}{B}(R) \backslash B(r) \longrightarrow \stackrel{\circ}{B}\left(\sqrt{R^{2}-r^{2}+\varepsilon^{2}}\right) \backslash B(\varepsilon)
$$

which yields, together with the above result $\ell_{e}\left(A^{2}\right)=\pi\left(R^{2}-r^{2}\right)$ that all $\mathcal{E}_{\omega}$-invariant capacities of $A^{2}$ are $\pi\left(R^{2}-r^{2}\right)$.

This example shows that $\mathcal{E}_{\omega}$-invariant capacities $C_{e}$ do not distinguish between annuli of the same area whereas $u$ does. On the other hand, $u$ does not distinguish between discs and annuli of the same (outer) radius, whereas $C_{e}$ might.

\section{Applications to closed characteristics and action inequalities.}

As $C_{H Z}(D) \leq u(D)$ for all $D$, one can draw a consequence of Theorem 4 in [HZ90]: If $u(D)$ is finite and $\partial D$ admits a foliation $S_{\varepsilon} \in[0,1]$ by hypersurfaces such that $S_{0}=\partial D$, then there exists a periodic solution on $S_{\varepsilon}$ for almost every $\varepsilon$ in $[0,1]$. This contains the almost existence theorem of Hofer and Zehnder in [HZ87] which generalized Viterbo's proof [V87] that every hypersurface of contact type carries at least one periodic orbit.

On the other hand, for a given $D$, the characterization of $c$ as a minimum of the dual Hamiltonian action functional together with Theorem 1(v) may be useful to show that $u(D)$ is finite.

For convex sets with $B(r) \subset K \subset B(R)$, a theorem by Croke-Weinstein and a theorem by Ekeland (see [E90] for both) state
(a) $\forall \gamma \in \Gamma(K) \quad A(\gamma) \geq \pi r^{2}$
(Croke-Weinstein)
(b) $\exists \gamma \in \Gamma(K) \quad A(\gamma) \leq \pi R^{2}$
(Ekeland)

These estimates can now be understood naturally in terms of capacities and are readily generalized:

Proposition 1. Consider $K \in \mathcal{K}$. If $D_{1} \subset K \subset D_{2}$ for two sets $D_{i} \in \mathcal{P}\left(\mathbb{R}^{2 n}\right)$, then for any extensions $C_{1}, C_{2}$ of $c$ one gets

(a) $\forall \gamma \in \Gamma(K) \quad A(\gamma) \geq C_{1}\left(D_{1}\right)$,

(b) $\exists \gamma \in \Gamma(K) \quad A(\gamma) \leq C_{2}\left(D_{2}\right)$.

Proof. Monotonicity and $C_{1}(K)=C_{2}(K)=c(K)$ imply

$$
C_{1}\left(D_{1}\right) \leq c(K)=\min _{\gamma \in \Gamma(K)} A(\gamma) \leq C_{2}\left(D_{2}\right)
$$


As concrete example, one can improve the inequalities already by taking for $D_{1}$ and $D_{2}$ two radially deformed ellipsoids [K90]. They are symplectomorphic to standard ellipsoids and have therefore known capacity.

4. Star-shaped domains need not be symplectomorphic to any convex set. Theorem 1 together with the definition of $c$ by closed characteristics on any set has an immediate

Corollary. Consider a subset $D_{0}$ of $\mathbb{R}^{2 n}$ with non-empty interior. Let $C\left(D_{0}\right)$ be its value for any symplectic capacity extending $c$. Then all sets $D \supset D_{0}$ carrying a characteristic loop on their boundary $\partial D$ with action strictly less than $C\left(D_{0}\right)$ cannot be symplectomorphic to a convex set. Consequently there are star-shaped domains which are not symplectomorphic to any convex set.

Proof. Assume $D=\varphi(K)$ for $K \in \mathcal{K}, \varphi \in \mathcal{D}_{\omega}$, and show that this leads to a contradiction. On the one hand

$$
C\left(D_{0}\right) \leq C(D)=C(\varphi(K))=c(K)=\inf \{A(\gamma) \mid \gamma \in \Gamma(K)\} ;
$$

but on the other, $\varphi$ induces a bijection between characteristic curves leaving the actions invariant, because $K$ and $\varphi(K)$ are simply connected, implying that for all characteristic loops on $\partial \varphi(K), A(\gamma) \geq c(K)=C(D)$, contradiction. For $C\left(D_{0}\right)=\infty$ the theorem means: If $\partial D$ carries a characteristic loop with finite action, then $D$ cannot be symplectically diffeomorphic to a convex set.

As examples, consider $D_{0}=B(r)$; then all sets $D \supset B(r)$ with a "neck loop" $\gamma$ as in the theorem are not symplectomorphic to a convex set. In particular, there are star-shaped domains which are not symplectomorphic to any convex set.

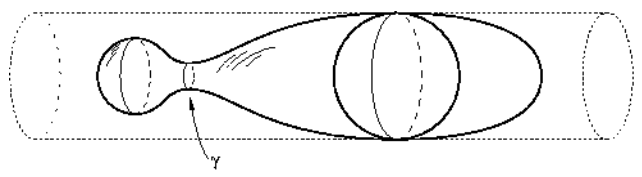

Fig. 2. A star-shaped domain which is not symplectomorphic to any convex set.

\section{Further examples.}

\section{Proposition 2}

(i) If $D \subset \mathbb{R}^{2 n-1} \subset \mathbb{R}^{2 n}$ is bounded, then $C(D)=0$ for all symplectic capacities $C$. For example $u\left(S^{2 n-2}\right)=0$, whereas $u\left(S^{2 n-1}\right)=u(B(1))=\pi$.

(ii) A Lagrangian plane $L$ satisfies $u(L)=\infty$.

(iii) Let $\stackrel{\circ}{D}_{1} \supset \bar{D}_{2}$, then $u\left(D_{1} \backslash D_{2}\right)=u\left(D_{1}\right)$.

(iv) Let $T^{d}=\partial B_{1} \times \cdots \times \partial B_{d}$ be a standard isotropic torus, where $B_{i}$ are simply connected 2-dimensional domains in the standard symplectic 2-space. Put $B_{i}=0, i=$ $d+1, \ldots, n$. Then $u\left(T^{d}\right)=\min _{i=1, \ldots, n} \operatorname{Area}\left(B_{i}\right)<\infty$ for all $d \leq n$ which is 0 for all $d<n$. Moreover, $C_{e}(\Lambda)=0$ for all $\mathcal{E}_{\omega}$-invariant capacities $C_{e}$ and for all Lagrangian tori $\Lambda$. 
(v) Let $\left\{D_{i} \mid i \in I\right\}$ be a collection of open bounded subsets with $\bar{D}_{i} \cap \bar{D}_{j}=\varnothing$ for $i \neq j$ and let $D=\bigcup_{i \in I} D_{i}$. Then $u(D) \geq \sup \left\{u\left(D_{i}\right)\right\} \geq \ell(D)$.

(vi) $u(\bar{D})=u(\stackrel{D}{D})$, but $\ell(\bar{D}) \neq \ell(\stackrel{D}{D})$ in general.

(vii) $u$ is Hausdorff-continuous on bounded domains, but $\ell$ is not.

This illustrates how much differently from measures capacities behave.

Proof.

(i) Consider a vector $e \in \mathbb{R}^{2 n}$ orthogonal to $D$ and $e^{\prime}=J e$ and the convex rectangle $K_{\varepsilon}:=[-R, R] e^{\prime} \times[-\varepsilon, \varepsilon] e \subset \operatorname{span}\left\{e^{\prime}, e\right\}=: E^{\perp} . D$ is contained in the symplectic product of convex sets $K_{\varepsilon} \times E$. By the product formula for $c$, one gets $C(D) \leq c\left(K_{\varepsilon} \times E\right)=$ $c\left(K_{\varepsilon}\right)=2 R \cdot 2 \varepsilon \rightarrow 0$ for $\varepsilon \rightarrow 0$.

This is true for any capacity, not only for extensions of $c$, because $K_{\varepsilon}$ is areapreserving diffeomorphic to a disc with area $2 \varepsilon=$ : $\pi r^{2}$, i.e. $K_{\varepsilon} \times E \sim B(r) \times \mathbb{R}^{2 n-2}$.

In conclusion, all bounded subsets of $\mathbb{R}^{2 n-1}$ have vanishing value for any capacity function $C$.

(ii) As $L$ is an $n$-dimensional plane in $\mathbb{R}^{2 n}$, its normal cone is an $n$-dimensional quadrant, whose image by $J_{0}$ is a quadrant in $L$. The differential inclusion $(*)$ has therefore no closed orbit, which means that $c(L)=\infty$.

(iii) $\varphi(K) \supset D_{1}$ if and only if $\varphi(K) \supset D_{1} \backslash D_{2}$ for $\stackrel{\circ}{D}_{1} \supset \bar{D}_{2}$, because $\varphi(K)$ is contractible. This implies $\mathcal{O}\left(D_{1} \backslash \bar{D}_{2}\right)=\mathcal{O}\left(D_{1}\right)$ and therefore $u\left(D_{1} \backslash D_{2}\right)=u\left(D_{1}\right)$. (Remark: A special case is the shell $\stackrel{\circ}{B}(R) \backslash B(r)$ we treated earlier.)

(iv) $T^{d} \subset \partial\left(\bigotimes_{i=1}^{n} B_{i}\right)=: \partial P$ where $P$ is the symplectic product of $B_{i}$ whose capacities can be estimated by the product formula for convex sets (with $B_{i}$ area-preserving diffeomorphic to convex discs):

$$
u(P)=\min \left\{u\left(B_{i}\right)\right\}=u\left(B_{k}\right),
$$

for some $k$. As $u\left(B_{k}\right)$ is the area of the bounded set $B_{k}, u\left(T^{d}\right)$ is bounded. If $d<n$, it is even 0 .

Now we can apply Moser's homotopy argument to show that all Lagrangian tori are symplectically equivalent, i.e. for all Lagrangian tori $\Lambda$, there is a $\varphi \in \mathcal{E}_{\omega}$ such that $\varphi(\Lambda)=T^{n}$ is a standard torus. Consequently

$$
C_{e}(\Lambda)=C_{e}(\varphi(\Lambda))=C_{e}\left(T^{n}\right) \leq u\left(B_{k}\right) .
$$

In particular, for all $\varepsilon>0$, there is a standard torus $T^{n}$ with $u\left(T^{n}\right)=\varepsilon$, i.e. $C_{e}(\Lambda)=0$ for all $\Lambda$ and $C_{e}$.

(v) $\varphi(K) \supset D \Rightarrow \varphi(K) \supset D_{i}: \mathcal{O}(D) \subset \mathcal{O}\left(D_{i}\right)$, implying $u(D) \geq \sup _{i \in I}\left\{u\left(D_{i}\right)\right\}$. If $\varphi(K) \subset D$, then it must be contained in one of the $D_{i}$ and conversely: $\mathcal{I}(D)=\bigcup_{i \in I} \mathcal{I}\left(D_{i}\right)$, yielding $\ell\left(\bigcup_{i \in I} D_{i}\right)=\sup _{i \in I}\left\{\ell\left(D_{i}\right)\right\}$.

(vi) For any symplectic diffeomorphism $\varphi$ defined on $\mathbb{R}^{2 n}$, one infers

$$
\stackrel{\circ}{D} \subset \varphi(K) \Longleftrightarrow \stackrel{\circ}{D} \subset \varphi(\stackrel{\circ}{K}) \Longleftrightarrow \bar{D} \subset \varphi(\bar{K}),
$$

from where $u(\bar{D})=u(\stackrel{D}{)}$.

(vii) Consider $D_{\varepsilon}=\left\{x \in \mathbb{R}^{2 n} \mid \operatorname{dist}(x, D) \leq \varepsilon\right\}$. Because $D_{\varepsilon}$ is bounded, the norm $\|d \varphi(x)\|$ is uniformly bounded from below and above on $D_{\varepsilon} \backslash D$. Then there exists a constant $r$ such that $u\left(D_{\varepsilon}\right)=(1+r \varepsilon) u(D)$, which proves the Hausdorff-continuity of $u$. 
Both negations for $\ell$ follow from the following counterexample: Consider a union $D=\bigcup_{i=1, \ldots, 4} D_{i}$ of four disjoint, juxtaposed open unit squares $D_{i}$ such that $\bar{D}$ is a closed square of length 2 . Then $\bar{D}$ has capacity $\ell(\bar{D})=4$, but $\ell(D)=\ell\left(D_{1}\right)=1$. Moreover $D_{\varepsilon} \supset \bar{D}$ for all $\varepsilon>0$.

THEOREM 2. For any capacity $C$ extending $c$ the generalized product formula holds:

(a) $\min \left\{\ell\left(D_{1}\right), \ell\left(D_{2}\right)\right\} \leq \ell\left(D_{1} \times D_{2}\right) \leq C\left(D_{1} \times D_{2}\right) \leq u\left(D_{1} \times D_{2}\right) \leq \min \left\{u\left(D_{1}\right), u\left(D_{2}\right)\right\}$.

(b) If $\ell\left(D_{i}\right)=u\left(D_{i}\right)$ for $i=1,2$, then $C\left(D_{1} \times D_{2}\right)=\min \left\{C\left(D_{1}\right), C\left(D_{2}\right)\right\}$.

Proof.

(a) Take a minimizing sequence $\left(K_{i}^{k}, \varphi_{i}^{k}\right), k \in \mathbb{N}$ for each $i$ and conclude: For $u$, assume $D_{i} \subset \varphi_{i}^{k}\left(K_{i}^{k}\right)$ and $u\left(D_{i}\right)=\inf _{k} c\left(K_{i}^{k}\right)$ for $i=1,2$. Clearly $D_{1} \times D_{2} \subset \varphi_{1}^{k}\left(K_{1}^{k}\right) \times$ $\varphi_{2}^{k}\left(K_{2}^{k}\right)$ and therefore using the product formula for convex sets $u\left(D_{1} \times D_{2}\right) \leq \inf _{k} c\left(K_{1}^{k} \times\right.$ $\left.K_{2}^{k}\right)=\inf _{k} \min \left\{c\left(K_{1}^{k}\right), c\left(K_{2}^{k}\right)\right\}=\min \left\{u\left(D_{1}\right), u\left(D_{2}\right)\right\}$, and similarly for $\ell$.

(b) follows immediately from (a)

Re mark. It is easy to see that there are 'many' sets satisfying the hypotheses of (b) which are not symplectomorphic to any convex set: Take for instance examples $D$ similar to the one in the Corollary to Theorem 1 such that moreover $B(r) \subset D \subset Z(r)$, see Figure 2. They all satisfy $\ell(D)=u(D)$ and are not symplectomorphic to any convex set, which shows that Theorem 2 is a true generalization of the product formula for $\mathcal{K}$.

Theorem 2 applies in particular to $c_{E H}$ (using [Si90]) and $c_{H Z}$.

6. Extensions to general symplectic manifolds. Now that extensions to $\mathbb{R}^{2 n}$ have been studied, it is easy to generalize them analogously to manifolds.

Definition 4. For any subset of a symplectic manifold of given dimension $2 n$, we define the non-negative numbers

$$
\begin{aligned}
\underline{u}(D) & =\inf _{\varphi \in \mathcal{E}_{\omega}} c(\operatorname{conv} \varphi(D)), \\
e(D) & =\sup \{c(K) \mid K \in \mathcal{K} \quad \text { such that } \exists \varphi \in \mathcal{S} \text { with } \varphi(K) \subset D\}, \\
k(D) & =\sup \{\underline{u}(P) \mid P \subset D \text { contractible }\} .
\end{aligned}
$$

THEOREM 3.

(i) $e, k$ and $\underline{u}$ satisfy the axioms of Definition 2 for any subsets of all symplectic manifolds and any family of embeddings.

(ii) All symplectic embedding capacities $C$ coinciding on $\mathcal{K}$ with $c$ are estimated by $e$ and $\underline{u}: e \leq C \leq \underline{u}$.

Proof. The proof is analogous to the one for $\ell$ and $u$ and is therefore skipped. For $k$, one simply observes that every $\varphi(K)$ is a contractible set, so that $e \leq k \leq \underline{u}$ immediately follows.

7. Surfaces. Given any compact surface $S$ of genus $g$, consider the canonical system of $2 g$ non-dividing curves $\alpha_{i}, \beta_{i}, i=1, \ldots, g$. Then $S \backslash A$ with $A:=\bigcup_{i=1}^{g} \alpha_{i} \cup \beta_{i}$ is 
conformally equivalent to a $2 g$-gon, which is itself conformally equivalent to a disk $D$ in $\mathbb{C}$ :

$$
f: S \backslash A \rightarrow D
$$

is a conformal map and is therefore symplectic:

$$
\operatorname{Area}(D)=\operatorname{Area}(S \backslash A)=\operatorname{Area}(S) .
$$

\section{Consequences.}

(1) $P=S \backslash A$ is contractible. Every other contractible subset of $S$ has area less than Area $(S)$, therefore $k(S)=\underline{u}(P)=\operatorname{Area}(S)$.

(2) $f^{-1}$ is a symplectic diffeomorphism $D \rightarrow S \backslash A$ from an open convex set into $S$, which realizes the maximum for area-preserving embeddings: $e(S)=\operatorname{Area}(S)$.

This proves

Proposition 3. For any surface $S$ with or without boundary, all symplectic embedding capacities $C$ extending $c$ are equal to the area of $S: e(S)=\operatorname{Area}(S)=k(S)$.

Proposition 3 has first been proved by Siburg [Si93] for embedding capacities (which he called Hofer-Zehnder capacities) by construction of an adapted Hamiltonian function.

This is in contrast to the diffeomorphism capacity $u$ which is different from the area: Recall that the annulus $S=B(R) \backslash \bar{B}(r)$ satisfies $e(S)=\operatorname{Area}(S)=k(S)=\pi\left(R^{2}-r^{2}\right)$ but $\underline{u}(S)=u(S)=\pi R^{2}$, see Figure 1 .

8. Symplectic 4-tori and the Herman-Zehnder example. Following [HZ94], we consider $\left(\mathbb{R}^{4}, \omega_{\alpha}\right)$ with the symplectic structure $\omega_{\alpha}(x, y)=A_{\alpha} X . Y$ defined by

$$
A_{\alpha}=\left(\begin{array}{cccc}
0 & -1 & \alpha_{2} & 0 \\
1 & 0 & -\alpha_{1} & 0 \\
-\alpha_{2} & \alpha_{1} & 0 & -1 \\
0 & 0 & 1 & 0
\end{array}\right)=-A_{\alpha}^{T}
$$

(which satisfies $\operatorname{det}\left(A_{\alpha}\right)=1$ but not $A_{\alpha}^{2}=-I$ ). This form induces a symplectic structure on the manifold $M=T^{3} \times[0, d]=\mathbb{R}^{3} / \mathbb{Z}^{3} \times[0, d]$ denoted again $\omega_{\alpha}$. For $\alpha_{1}, \alpha_{2}=0$, one gets the standard almost complex structure $J_{0}$. For $d<1,\left(M, \omega_{\alpha}\right)$ is embedded in the torus $\left(T^{4}, \omega_{\alpha}\right)$.

Functions $H$ on $\mathbb{R}^{4}$ which are 1-periodic in the first three variables pass to the quotient as well as their Hamiltonian vector fields

$$
\xi_{H}:=-A_{\alpha}^{-1} H^{\prime}(x),
$$

where $H^{\prime}(x)$ is the Euclidean gradient of $H$. As

$$
A_{\alpha}^{-1}=\left(\begin{array}{cccc}
0 & -1 & 0 & -\alpha_{1} \\
1 & 0 & 0 & -\alpha_{2} \\
0 & 0 & 0 & -1 \\
\alpha_{1} & \alpha_{2} & 1 & 0
\end{array}\right),
$$

we get for the Hamiltonian function $H_{0}(x)=x_{4}$ a constant vector field

$$
\xi_{H_{0}}=\left(\alpha_{1}, \alpha_{2}, 1,0\right)=:(\alpha, 0),
$$


which integrates to an affine flow preserving all 3 -tori $T^{3} \times\{s\}$. If $\alpha=\left(\alpha_{1}, \alpha_{2}, 1\right)$ is rationally independent, i.e. $\alpha . z \neq 0 \quad \forall z \in \mathbb{Z}^{3} \backslash 0$, this flow is dense and has no periodic orbits. Therefore it represents an example of a Hamiltonian flow whose energy levels $T^{3} \times\{s\}$ are all regular and compact but none of them carries a periodic orbit.

M. Herman proved in [H91] that $H_{0}$ is dynamically stable under perturbations if $\alpha$ is irrational satisfying a diophantine condition: This represents an counter-example against the $C^{k}$-closing conjecture for $k$ sufficiently large.

In [HZ94], it has been showed that $c_{H Z}\left(M, \omega_{\alpha}\right)$ is infinite if $\alpha$ is irrational. Here we estimate $C\left(M, \omega_{\alpha}\right)$ for any $C$ extending $c$ by exhibiting a convex set contained in $M$ :

$$
C\left(M, \omega_{\alpha}\right) \begin{cases}=\infty & \text { if } \alpha \text { irrational } \\ \geq d & \text { if } \alpha \text { rational }\end{cases}
$$

Pro of. Consider the linear map $\left(\mathbb{R}^{4}, \omega\right) \rightarrow\left(\mathbb{R}^{4}, \omega_{\alpha}\right)$ given by the matrix

$$
N_{\alpha}=\left(\begin{array}{cccc}
1 & 0 & \alpha_{1} & 0 \\
0 & 1 & \alpha_{2} & 0 \\
0 & 0 & 1 & 0 \\
0 & 0 & 0 & 1
\end{array}\right)
$$

which is symplectic: $N_{\alpha}^{t} A N_{\alpha}=J_{0}$. Denote the canonical basis by $e_{k}$.

(i) If $\alpha$ is irrational, then the Lagrangian plane $L$ spanned by $e_{1}$ and $e_{3}$ is embedded by $N_{\alpha}$ into $\tilde{M}=\mathbb{R}^{3} \times[0, d]$. The quotient of this map onto $M$ winds $L$ in the 3 -torus densely around itself. But a Lagrangian plane has infinite capacity, from where the first part of the claim.

If $\alpha$ is rational, then there are relatively prime $n_{i} \in \mathbb{Z}, i=1,2$ such that $\alpha_{i}=\frac{n_{i}}{n_{3}}$. Then:

(ii) $N_{\alpha}$ embeds the standard unit 3-cube into a fundamental domain of the action of $\mathbb{Z}^{3}$ on $\tilde{M}$. Therefore $C\left(M, \omega_{\alpha}\right) \geq c\left([0,1]^{3} \times[0, d], \omega_{0}\right)=d$ by the product formula, which proves the second claim.

(iii) But the map $N_{\alpha}$ also sends then the parallelogram $P$ spanned by $e_{1}, \frac{1}{n_{3}} e_{2}, n_{3} e_{3}, e_{4}$ into a fundamental domain of the action of $\mathbb{Z}^{3}$ on $\tilde{M}$. Therefore $C\left(M, \omega_{\alpha}\right) \geq c\left(P, \omega_{0}\right)$, which is equal to $\min \left\{\frac{1}{n_{3}}, d\right\}$ again by the product formula for $c$.

This last observation shows the relation to (i), but also prompts a question concerning fundamental domains of in $\tilde{M}$ (which would determine $e(M)$ ): What is the biggest capacity a fundamental domain in $\tilde{M}$ can have?

\section{References}

[A84] J. P. Aubin, L'analyse non linéaire et ses motivations économiques, Masson, Paris, 1984.

[CE80] F. H. Clarke, I. Ekeland, Hamiltonian trajectories with prescribed minimal period, Comm. Pure Appl. Math. 33 (1980), 103-116.

[E90] I. Ekeland, Convexity methods in Hamiltonian mechanics, Springer, Berlin-Heidelberg, 1990. 
[EH89] I. Ekeland, H. Hofer, Symplectic Topology and Hamiltonian Dynamics I, Math. Z. 200 (1989), 355-378. See also C. R. Acad. Sci. Paris Sér. I Math. 307 (1988), 37-40.

[EH90] I. Ekeland, H. Hofer, Symplectic Topology and Hamiltonian Dynamics II, Math. Z. 203 (1990), 553-567.

[G85] M. Gromov, Pseudoholomorphic curves in symplectic manifolds, Invent. Math. 82 (1985), 307-347.

[H91] M. Herman, Exemples de flots Hamiltoniens dont aucune perturbation en topologie $C^{\infty}$ n'a d'orbites périodiques sur un ouvert de surfaces d'énergies, C. R. Acad. Sci. Sér. I Math. 312 (1991), 989-994.

[HZ87] H. W. Hofer, E. Zehnder, Periodic solutions on hypersurfaces and a result by $C$. Viterbo, Invent. Math. 90 (1987), 1-9.

[HZ90] H. W. Hofer, E. Zehnder, A new capacity for symplectic manifolds, in: Analysis et cetera, Academic Press, 1990, 405-429.

[HZ94] H. Hofer, E. Zehnder, Symplectic Invariants and Hamiltonian Dynamics, Birkhäuser, Basel-Boston-Berlin, 1994.

[K90] A. F. Künzle, Une capacité symplectique pour les ensembles convexes et quelques applications, Ph.D. thesis, Université Paris IX Dauphine, June 1990.

[K91] A. F. Künzle, The least characteristic action as symplectic capacity, preprint, Forschungsinstitut für Mathematik, ETH Zürich, May 1991.

[K93] A. F. Künzle, Singular Hamiltonian systems and Symplectic Capacities, in: Singularities and Differential Equations, Banach Center Publ. 33 (1996), 171-187.

[R70] R. T. Rockafellar, Convex Analysis, Princeton University Press, Princeton N.J., 1970.

[Si93] K. F. Siburg, Symplectic capacities in two dimensions, Manuscripta Math. 78 (1993), 149-163.

[Si90] J. C. Sikorav, Systèmes Hamiltoniens et topologie symplectique, Lecture Notes, Dipartimento di Matematica dell'Università di Pisa, August 1990.

[V87] C. Viterbo, A proof of the Weinstein conjecture in $\mathbb{R}^{2 n}$, Ann. Inst. H. Poincaré Anal. Non Linéaire 4 (1987), 337-357. 